\title{
The effect of omalizumab treatment on severe allergic asthma and allergic comorbidities: real-life experience from the Czech Anti-IgE Registry
}

\author{
Beáta Hutyrová1,2, Jaromír Bystroñ ${ }^{1}$; Czech Anti-lgE Registry collaborators \\ 1Department of Allergology and Clinical Immunology, University Hospital Olomouc, Olomouc, Czech Republic \\ 2Department of Immunology, Faculty of Medicine and Dentistry, Palacký University, Olomouc, Czech Republic
}

Adv Dermatol Allergol 2018; XXXV (5): 510-515 DOI: https://doi.org/10.5114/ada.2018.77243

\begin{abstract}
Introduction: Omalizumab is indicated for the treatment of severe allergic asthma (SAA) and chronic spontaneous urticaria, although a number of studies have confirmed the effectiveness of this therapy also for other IgE-mediated diseases.

Aim: To assess the impact of anti-lgE therapy on SAA and comorbid IgE-mediated allergic diseases in patients treated with omalizumab for SAA enrolled in the CAR (Czech Anti-lgE Registry).

Material and methods: Three hundred and ten patients with SAA treated with omalizumab were enrolled in the CAR. Two hundred and twenty-nine individuals were evaluated after 12 months of omalizumab treatment for asthma control test (ACT), examination of fractional exhaled nitric oxide (FENO), forced expiratory volume in $1 \mathrm{~s}$ $\left(\mathrm{FEV}_{1}\right)$, the use of systemic corticosteroids, side effects of treatment and clinical effect of omalizumab on allergic comorbidities (allergic rhinitis, chronic urticaria, atopic dermatitis and food allergy).

Results: After 12 months of treatment with omalizumab, patients experienced a significant improvement of ACT and $\mathrm{FEV}_{1}$, reduction of FENO, use of systemic corticosteroids for asthma exacerbations and dose of maintenance oral corticosteroid therapy. The positive effect of treatment with omalizumab was observed in $82.2 \%$ of patients with allergic rhinitis, in $85.7 \%$ of patients with chronic urticaria, in $82.1 \%$ of patients with atopic dermatitis, and in $67.3 \%$ of patients with food allergy.

Conclusions: In the CAR registry, patients with SAA treated with omalizumab showed a significant positive effect of anti-lgE therapy not only on the asthma control, but also on allergic comorbidities.
\end{abstract}

Key words: omalizumab, severe allergic asthma, allergic diseases

\section{Introduction}

Omalizumab (Novartis, Switzerland; Genentech, USA) is a recombinant humanised IgG1 monoclonal antibody that selectively binds to the $\mathrm{CH} 3$ domain of free immunoglobulin $\mathrm{E}$ ( IgE) and prevents it from binding to the high affinity receptor Fc\&RI on the cell membrane of mast cells, basophils and antigen presenting cells $[1,2]$. This leads to reduction in free serum IgE, down-regulation of FceRI on the cell membrane of key inflammatory cells with a consequent blockade of the allergic cascade, antigen presentation to T-lymphocytes, production of Th2 cytokines and other mediators, reduction in the number of eosinophils in peripheral blood and tissues [1, 2]. These mechanisms ultimately reduce a pathological allergic immune response and inflammation.

Omalizumab is indicated for treatment of severe allergic asthma (SAA) and chronic spontaneous urticaria, but several studies confirm a positive effect of this therapy also on other IgE-mediated allergic diseases [3, 4]. The use of omalizumab in the treatment of other allergic diseases is still considered to be an off-label therapy.

\section{Aim}

The aim of this study was to evaluate the effect of anti-lgE therapy with omalizumab on SAA and other allergic comorbidities (allergic rhinitis, chronic urticaria,

Address for correspondence: Beáta Hutyrová MD, PhD, Department of Allergology and Clinical Immunology, University Hospital Olomouc, 6 I. P. Pavlova St, 77520 Olomouc, Czech Republic, phone: +42 0588444791, e-mail: beata.hutyrova@fnol.cz Received: 13.06.2017, accepted: 11.09.2017. 
atopic dermatitis, food allergy) in the group of patients treated with omalizumab for SAA.

\section{Material and methods \\ Patients}

Three hundred and ten patients with SAA treated with omalizumab as a part of a normal clinical practice were enrolled in the Czech Anti-IgE Registry (CAR) in 10 specialized centers in the Czech Republic between 2006 and 2015. The study was designed as a retrospective-prospective, multicenter, non-interventional, observational study. The patients, enrolled in the CAR, were retrospectively evaluated for clinical conditions in the last year before the initiation of the omalizumab therapy. All patients fulfilled GINA criteria for severe asthma, their asthma was classified as allergic with proven allergy to perennial airborne allergens, and had $\geq 2$ moderate to severe asthma exacerbations in the last year before the initiation of the omalizumab treatment [5]. The patients were evaluated by physicians after 16 weeks of treatment with omalizumab as responders or non-responders on the basis of global evaluation of asthma control. The therapy with omalizumab was discontinued in the non-responders.

From a total of 310 patients enrolled in the CAR, 229 individuals were evaluated after 12 months of administration of omalizumab. The following was assessed after 12 months of the treatment: asthma control (asthma control test $-A C T^{T M}$ ), examination of fractional exhaled nitric oxide (FENO), forced expiratory volume in $1 \mathrm{~s}$ $\left(F E V_{1}\right)$, the use of systemic corticosteroids (maintenance treatment and bursts of corticosteroids due to asthma exacerbations), side effects of the treatment and clinical effect of omalizumab on allergic comorbidities (allergic rhinitis, chronic urticaria, atopic dermatitis and food allergy). Evaluation of comorbidities has been carried out on the basis of history and subjective assessment of the patient's physician. The course of disease was assessed by four categories: 1) free of symptoms, 2) improvement, 3) no change, and 4) worsening of symptoms. Not all of the followed parameters were available in all patients.

\section{Statistical analysis}

Categorical variables are described by an absolute and relative frequency in the groups of patients. For the description of the continuous variables, methods of descriptive statistics with calculation of average, median and range (minimum-maximum) were used. The Wilcoxon paired test was used for a comparison of continuous variables. The level of statistical significance was considered to be $p<0.05$. The analysis was performed by the statistical program SPSS 22.0.0 (IBM Corporation, 2013).

\section{Results}

\section{Patient baseline characteristics}

A total of 310 patients were enrolled in the study. Overall, 187 (60.3\%) patients were women and 123 (39.7\%) patients were men. The average age of the patients at baseline was 44 years (median: 43 years, range: $8-78$ years). The average value of total IgE levels was $350 \mathrm{IU} /$ $\mathrm{ml}$, median $239 \mathrm{lU} / \mathrm{ml}$ and range 16-2303 IU/ml. Two hundred and twenty-eight patients $(75.0 \%, n=304)$ received maintenance oral corticosteroid (OCS) therapy, and the average daily OCS dose (prednisone) was $10.2 \mathrm{mg}$ (median: $5 \mathrm{mg}$, range: $0-100 \mathrm{mg}$ ) at baseline. The patients were dosed 150-1200 mg/month of omalizumab according to their weight and IgE levels. The mean values of FEV 1 were 66\% (median: 67\%, range: $10-120 \%, n=309$ ) at baseline. Distribution of $\mathrm{FEV}_{1}$ values among patients was the following: > 80\% - 60 (19.4\%) patients, 60-80\% - 140 (45.3\%) patients, $40-60 \%-77$ (24.9\%) patients, < 40\% - 32 (10.4\%) patients. Baseline FENO values were available in $221 \mathrm{in-}$ dividuals with mean $51 \mathrm{ppb}$, median $37 \mathrm{ppb}$ and range 0-290 ppb. There were measured FENO values $\geq 25 \mathrm{ppb}$ in $80(36.2 \%)$ patients, $25-50$ ppb in 62 (28.1\%) patients, 51-75 ppb in 34 (15.4\%) patients, 76-100 ppb in 14 (6.3\%) patients, and > $100 \mathrm{ppb}$ in 31 (14.0\%) patients. The mean ACT was 12 points (median: 12 points, range: $5-24$ ) at baseline $(n=295)$. Initially, in $269(86.8 \%)$ patients allergic rhinitis, in $86(27.7 \%)$ patients atopic dermatitis, in 81 (26.1\%) patients food allergy, and in 17 (5.5\%) patients chronic urticaria were reported $(n=310)$.

\section{Effect of omalizumab on asthma}

From a total of 310 patients enrolled, 52 (16.7\%) patients discontinued the therapy after 16 weeks because they were identified as non-responders by their physicians. The values of ACT, FEV and FENO at baseline and 12 months after the initiation of omalizumab therapy are presented in Table 1 . After 12 months of treatment with omalizumab, a significant improvement in ACT score,

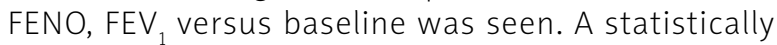
significant reduction in the use of systemic corticosteroids for asthma exacerbation was found from baseline to month 12 ( $p<0.001)$ (Figure 1). After 12 months of the omalizumab treatment, a daily OCS dose was significantly decreased (mean $5 \mathrm{mg}$, median $0 \mathrm{mg}$, range: 0-65 mg after 12 months vs. mean $10.2 \mathrm{mg}$, median $5 \mathrm{mg}$, range: $0-100 \mathrm{mg}$ at baseline, $n=224, p<0.001)$. Ninety-two (41.1\%) patients discontinued OCS treatment during 12 months of omalizumab therapy.

\section{Effect of omalizumab on concomitant allergic diseases}

Evaluation of the subgroup of 229 patients who followed the therapy for 12 months has shown that allergic rhinitis was present in 203 (88.6\%) patients, atopic dermatitis in 67 (29.2\%) patients, food allergy in 58 (25.3\%) 
Table 1. Change of ACT score, FENO and FEV ${ }_{1}$ after 12 months of omalizumab therapy

\begin{tabular}{|c|c|c|c|c|c|c|}
\hline Parameter & $N^{*}$ & Therapy & Mean & Median & Range & $P$-value \\
\hline \multirow[t]{2}{*}{ ACT } & 214 & Baseline & 13 & 12 & $5-23$ & $<0.001$ \\
\hline & & Month 12 & 18 & 19 & $5-25$ & \\
\hline \multirow[t]{2}{*}{$\mathrm{FEV}_{1}$} & 224 & Baseline & $65 \%$ & $67 \%$ & $10-120 \%$ & $<0.001$ \\
\hline & & Month 12 & $73 \%$ & $74 \%$ & $12-128 \%$ & \\
\hline \multirow[t]{2}{*}{ FENO } & 138 & Baseline & $50 \mathrm{ppb}$ & $36 \mathrm{ppb}$ & $0-272 \mathrm{ppb}$ & 0.003 \\
\hline & & Month 12 & $37 \mathrm{ppb}$ & $28 \mathrm{ppb}$ & 0-341 ppb & \\
\hline
\end{tabular}

${ }^{*}$ Calculated only for patients with information about the parameter at both time points.

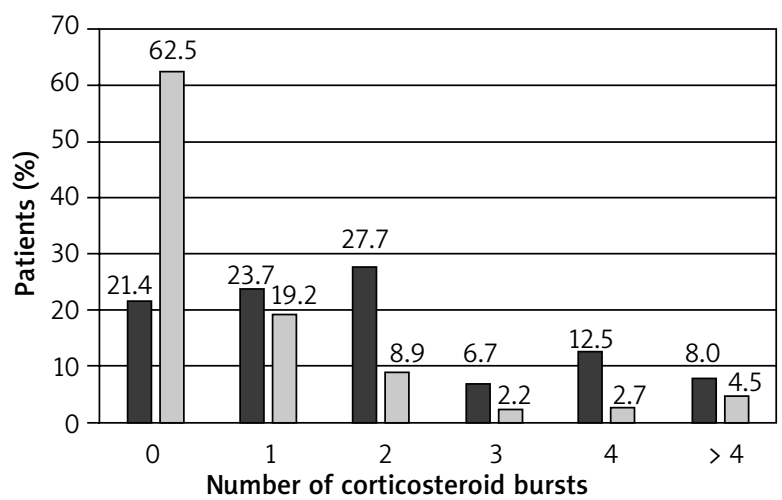

$\square$ Before treatment $\quad \square$ After 12 months of treatment

Figure 1. Use of systemic corticosteroid bursts for asthma exacerbation at baseline and after 12 months of omalizumab treatment $(n=224, p<0.001)$

patients, and chronic urticaria in 14 (6.1\%) patients. After 12 months of omalizumab treatment, the symptoms of allergic rhinitis disappeared in 38 (18.7\%) patients, an improvement was reported in 129 (63.5\%) patients, symptoms worsened in 1 (0.5\%) patient, and in 32 (15.8\%) patients it remained unchanged. Atopic dermatitis completely resolved in 24 (35.8\%) patients, improved in 31 (46.3\%) patients, remained unchanged in 9 (13.4\%) patients and worsened in $2(3.0 \%)$ patients. Complete remission of food allergy was reported in 23 (39.7\%) patients, improvement in 16 (27.6\%) patients and in 18 (31.0\%) patients, symptoms remained unchanged. None of the patients with food allergy reported worsening of symptoms. In 5 (35.7\%) patients, urticaria disappeared during the treatment with omalizumab and it improved in 7 (50.0\%) patients, in 1 patient the symptoms were unchanged and in 1 (7.1\%) patient the symptoms worsened. The results of assessments of omalizumab treatment's effect on these comorbidities are shown in Table 2.

\section{Adverse effects of omalizumab}

Adverse events occurred in $38(12.9 \%, n=295)$ and 16 $(7.0 \%, n=229)$ patients after 4 and 12 months of therapy, respectively. The most common adverse effects were headache, nausea, dysphonia, fatigue, weight gain and paresthesia. In 11 (3.5\%) patients, adverse effects resulted in discontinuation of therapy with omalizumab. There were no serious adverse events.

\section{Discussion}

In the present study we have evaluated the effect of omalizumab in patients with severe allergic asthma included in the CAR registry after 12 months of treatment. It is a well-documented fact that treatment with anti-lgE monoclonal antibody provides significant benefits for patients with SAA [6]. This treatment significantly improves symptoms and quality of life, it reduces the rate of asthma exacerbations, asthma hospitalizations and doses of systemic and inhaled corticosteroids [7]. The results of this study have confirmed a significant improvement of asthma control (measured by ACT) and respiratory functions ( $\mathrm{FEV}_{1}$ ), significant decrease of FENO and overall use of systemic corticosteroids (maintenance corticosteroid treatment and corticosteroid bursts due to asthma exacerbations) after 12 months of treatment with omalizumab. The therapy with omalizumab was discontinued in $16.7 \%$ of enrolled patients, who were identified as

Table 2. Change of symptoms of allergic comorbidities after 12 months of omalizumab treatment

\begin{tabular}{lcccccc}
\hline Symptoms & $\boldsymbol{N}$ & Asymptomatic & Improvement & No change & Worsening & NA \\
\hline Allergic rhinitis & 203 & $18.7 \%(38)$ & $63.5 \%(129)$ & $15.8 \%(32)$ & $0.5 \%(1)$ & $1.5 \%(3)$ \\
\hline Atopic dermatitis & 67 & $35.8 \%(24)$ & $46.3 \%(31)$ & $13.4 \%(9)$ & $3 \%(2)$ & $1.5 \%(1)$ \\
\hline Food allergy & 58 & $39.7 \%(23)$ & $27.6 \%(16)$ & $31 \%(18)$ & 0 & $1.7 \%(1)$ \\
\hline Chronic urticaria & 14 & $35.7 \%(5)$ & $50 \%(7)$ & $7.1 \%(1)$ & $7.1 \%(1)$ & 0 \\
\hline
\end{tabular}

NA - not available. 
non-responders by their physicians after 16 weeks of treatment. The therapy was well tolerated and only in $3.5 \%$ of patients the treatment was terminated due to adverse effects.

Apart from assessing the asthma control, we have also focused on the effect of omalizumab on comorbidities of allergic etiology - allergic rhinitis, atopic dermatitis, food allergy and chronic urticaria. Omalizumab is nowadays approved also for the treatment of chronic spontaneous urticaria, but the effectiveness of omalizumab has also been studied in other diseases [4, 7]. The most common comorbidity in our group of SAA patients was allergic rhinitis present in $86.8 \%$ of individuals. The positive effect of treatment with omalizumab was observed in $82.2 \%$ of patients with allergic rhinitis after 12 months of therapy (improvement in $63.5 \%$ of patients, complete remission in $18.7 \%$ of patients). The efficacy of omalizumab on allergic rhinitis has been shown in numerous studies. Meta-analysis of 11 studies involving 2,870 patients with moderate-to-severe allergic rhinitis, who were treated with omalizumab due to an inadequate compensation of the disease despite the standard treatment, showed a statistically significant reduction in the daily nasal symptom severity score $(-0.67$, $p<0.0001)$, in daily nasal rescue medication score and improvement of quality of life [8].

In patients with severe asthma and periodic allergic rhinitis treated with omalizumab, there has been shown a reduction of symptoms (nasal obstruction, rhinorrhea, itching, sneezing), resolution of turbinate hypertrophy, reduction or elimination of symptomatic drug use [9]. Series of randomized, double-blind, placebo-controlled (RDBPC) multicenter studies have shown that the combination of allergen-specific immunotherapy (SIT) with omalizumab significantly improves the tolerance, safety and efficacy of SIT in patients with allergic rhinitis and asthma $[1,10,11]$.

Other comorbidities, followed in patients enrolled in the CAR registry, were chronic urticaria and atopic dermatitis. In our patients with SAA and chronic urticaria, efficacy of omalizumab after 12 months of treatment was seen in $85.7 \%$ of patients (improvement in $50 \%$ of patients, complete resolution in $35.7 \%$ of patients), which corresponds with the literature data. Chronic spontaneous urticaria is the only skin disease, for which omalizumab therapy is currently approved. The effectiveness of antiIgE therapy for this diagnosis has been demonstrated in several RDBPC studies involving almost 1,200 patients $[12,13]$. In these studies, different doses of omalizumab ranging from $75 \mathrm{mg}$ to $600 \mathrm{mg}$ with treatment duration up to 24 weeks were examined. $300 \mathrm{mg}$ of omalizumab administered every 4 weeks has shown to be the most effective and it resulted in a significant reduction of symptoms (pruritus), number of urticarial wheals, it improved the quality of life of patients and decreased the dose of antihistamines. Complete resolution of urticaria was reported in 34-44\% of patients, complete or nearly complete resolution of the symptoms was observed in $52-66 \%$ of patients treated with 300 mg of omalizumab every 4 weeks [12].

The role of omalizumab in the treatment of atopic dermatitis is not as clear as in chronic spontaneous urticaria. Although atopic dermatitis is associated with high serum IgE levels, the role of IgE in pathogenesis of this disease has not been completely explained. Atopic dermatitis occurs even in patients without any evidence of atopy, in which non-lgE-mediated pathogenetic mechanisms occur. In our patients from the CAR registry, an improvement of atopic dermatitis was reported in $46.3 \%$ of patients and $35.8 \%$ of patients had no symptoms of skin disease after 12 months of treatment with omalizumab (overall positive effect in $82.1 \%$ of patients). Published studies with anti-IgE treatment in atopic dermatitis reported ambivalent results. Some of them have shown the benefit of treatment with omalizumab in atopic dermatitis [14-16], while others have not confirmed its efficacy $[17,18]$. Our data are similar to results of a recent meta-analysis of 26 studies encompassing 174 patients with atopic dermatitis. This meta-analysis reported positive clinical effects of omalizumab ranging from mild improvement to complete remission in $74.1 \%$ of patients [19]. Another meta-analysis of 15 studies investigating the effect of omalizumab in atopic dermatitis discovered an excellent clinical response in $43 \%$ of patients, $27.2 \%$ had a satisfactory response and in $30.1 \%$ of patients there were no changes or symptoms worsened [20]. Multivariate logistic regression showed that an excellent clinical response was significantly associated with serum IgE levels of less than $700 \mathrm{IU} / \mathrm{ml}$ compared to patients with high serum IgE concentrations. A possible reason for the failure of anti-lgE therapy in these studies is the low dose of omalizumab that "inactivates" the IgE by immune complex formation and depends on the ratio of molecules of omalizumab and IgE [20, 21]. A new approach in the treatment of atopic dermatitis with very high IgE serum levels could therefore be a combination of extracorporeal IgE immunoadsorption with anti-lgE monoclonal antibody [21]. This treatment was applied in the study of Zink et al. in 10 patients with severe atopic dermatitis with serum IgE > $3500 \mathrm{kU} / \mathrm{l}$. Patients underwent the immunoadsorption within 2 to 4 consecutive days, depending on the baseline level of total IgE, followed by an administration of $450 \mathrm{mg}$ of omalizumab every 2 weeks for 24 weeks. In all patients, the levels of free IgE decreased with the improvement of symptom severity score [21]. Another reason for lack of efficacy of therapy with omalizumab in atopic dermatitis might be complex immune dysregulation caused by non-IgE-mediated pathogenic mechanisms. Patients with the primary disorder of the skin barrier with filaggrin gene mutations are less likely to benefit from treatment with omalizumab compared with patients with mutations in other genes [22]. 
From all the allergic comorbidities in our SAA patients, the least efficacy of omalizumab therapy was observed in patients with food allergy. Positive effects of omalizumab were seen in $67.3 \%$ of patients after 12 months of treatment. $27.6 \%$ of patients reported improvement and $39.7 \%$ of patients had complete remission of symptoms of food allergy. In comparison with other monitored diseases, there was the highest proportion of individuals (31\%) with no change in symptoms during treatment with omalizumab in patients with food allergy. A possible explanation could be the ineffectiveness of omalizumab with food allergy mediated by non-IgE mechanisms. So far no effective treatment of food allergies was available except for elimination diet and symptomatic treatment of allergic reactions. In recent years, the possibility of using oral immunotherapy (OIT) to achieve tolerance to food allergens was studied [23-27]. Omalizumab is one of the most promising adjunctive therapies for food allergy. The allergens studied in omalizumab-enabled OIT trials include peanuts, cow's milk, eggs, or mixes of multiple foods [25]. Results from several studies suggest that omalizumab allows the use of more efficient rapid dose-escalation protocols for OIT. Omalizumab reduces the frequency and severity of adverse reactions induced by OIT, and increases the allergen tolerance threshold [25-27].

\section{Conclusions}

Data from the Czech Anti-IgE Registry confirm the "real-life experience" from other countries with omalizumab treatment in patients with SAA. In the present study, a significant improvement in asthma control, lung functions, reduction of exacerbations and overall use of systemic corticosteroid were demonstrated after 12 months of omalizumab treatment. Furthermore, positive effects of omalizumab have been proven on allergic comorbidities in the majority of patients with SAA. Most patients with allergic rhinitis, atopic dermatitis, chronic urticaria and food allergy experienced improvement or complete remission of symptoms of these diseases. Except for SAA and chronic spontaneous urticaria, the use of omalizumab in the treatment of other allergic diseases is still considered off-label therapy. Patients with severe forms of other allergic diseases, inadequately controlled with standard therapies, may also significantly benefit from omalizumab treatment. Therefore, it is necessary to verify the efficacy of omalizumab in further RDBPC studies with larger groups of patients with allergic diseases. As the results are ambiguous in some disorders, defining subgroups of responders and finding predictors of therapeutic response to omalizumab may be useful in these diseases.

\section{Acknowledgments}

The authors would like to acknowledge the following collaborators for their contributions to the CAR:
Dr. Jan Chlumský, Dr. Dagmar Kindlová, Dr. Jana Klosová, Dr. Irena Krčmová, Dr. Bronislava Novotná, Professor Petr Panzner, Professor Petr Pohunek, Dr. Patricie Popelková, Dr. Olga Růžičková-Kirchnerová, Dr. Vratislav Sedlák, Dr. Irena Starová, Associate Professor Dr. Milan Teřl, Dr. Petr Vaník, Professor Martina Vašáková, Dr. Vernerová Eva, Dr. Jaromir Zatloukal. The authors thank the Institute of Biostatistics and Analyses at the Faculty of Medicine and the Faculty of Science of the Masaryk University Brno, Czech Republic (IBA MU) for administration of the CAR database and statistical analysis.

\section{Funding}

This study was funded by Novartis s.r.o.

\section{Conflict of interest}

The authors declared the following potential conflicts of interest: Associate Professor Jaromír Bystroň participated in advisory boards or received consultancy/lecture fees from Novartis, Teva, ALK, Stallergenes. Beáta Hutyrová declares no conflict of interest.

\section{References}

1. Ciprandi G, Marseglia GL, Castagnoli R, et al. From IgE to clinical trials of allergic rhinitis. Expert Rev Clin Immunol 2015; 11: 1321-33.

2. Mitchell PD, El-Gammal AI, O‘Byrne PM. Emerging monoclonal antibodies as targeted innovative therapeutic approaches to asthma. Clin Pharmacol Ther 2016; 99: 38-48.

3. Landolina N, Levi-Schaffer F. Monoclonal antibodies: the new magic bullets for allergy: IUPHAR Review 17. Br J Pharmacol 2016; 173: 793-803.

4. El-Qutob D. Off-label uses of omalizumab. Clin Rev Allergy Immunol 2016; 50: 84-96.

5. Global Strategy for Asthma Management and Prevention (2017 Update). http://ginasthma.org/2017-gina-reportglobal-strategy-for-asthma-management-and-prevention/.

6. Fajt ML, Wenzel SE. Development of new therapies for severe asthma. Allergy Asthma Immunol Res 2017; 9: 3-14.

7. Incorvaia C, Mauro M, Russello M, et al. Omalizumab, an anti-immunoglobulin E antibody: state of the art. Drug Des Devel Ther 2014; 8: 197-207.

8. Tsabouri S, Tseretopoulou X, Priftis K, Ntzani EE. Omalizumab for the treatment of inadequately controlled allergic rhinitis: a systemic review and meta-analysis of randomized clinical trials. J Allergy Clin Immunol 2014; 2: 332-40.

9. Masieri S, Cavaliere C, Begvarfaj E, et al. Effects of omalizumab therapy on allergic rhinitis: a pilot study. Eur Rev Med Pharmacol Sci 2016; 20: 5249-55.

10. Kopp MV, Hamelmann E, Zielen S, et al. Combination of omalizumab and specific immunotherapy is superior to immunotherapy in patients with seasonal allergic rhinoconjunctivitis and co-morbid seasonal allergic asthma. Clin Exp Allergy 2009; 39: 271-9.

11. Kamin W, Kopp MV, Erdnuess F, et al. Safety of anti-IgE treatment with omalizumab in children with seasonal allergic rhinitis undergoing specific immunotherapy simultaneously. Pediatr Allergy Immunol 2010; 21: 160-5. 
12. Vestergaard C, Deleuran M. Chronic spontaneous urticaria: latest developments in aetiology, diagnosis and therapy. Ther Adv Chronic Dis 2015; 6: 304-13.

13. Maurer M, Rosén K, Hsieh HJ, et al. Omalizumab for the treatment of chronic idiopathic or spontaneous urticaria. N Engl J Med 2013; 368: 924-35.

14. Sheinkopf LE, Rafi AW, Do LT, et al. Efficacy of omalizumab in the treatment of atopic dermatitis: a pilot study. Allergy Asthma Proc 2008; 29: 530-7.

15. Belloni B, Ziai M, Lim A, et al. Low-dose anti-lgE therapy in patients with atopic eczema with high serum IgE levels. J Allergy Clin Immunol 2007; 120: 1223-5.

16. Kim DH, Park KY, Kim BJ, et al. Antiimmunoglobulin E in the treatment of refractory atopic dermatitis. Clin Exp Dermatol 2013; 38: 496-500.

17. Heil PM, Maurer D, Klein B, et al. Omalizumab therapy in atopic dermatitis: depletion of IgE does not improve the clinical course - randomized, placebo-controlled and doubleblind pilot study. J Dtsch Dermatol Ges 2010; 8: 990-8.

18. Krathen RA, Hsu S. Failure of omalizumab for treatment of severe adult atopic dermatitis. J Am Acad Dermatol 2005; 53: 338-40.

19. Holm JG, Agner T, Sand C, Thomsen SF. Omalizumab for atopic dermatitis: case series and a systematic review of the literature. Int J Dermatol 2017; 56: 18-26.

20. Wang HH, Li YC, Huang YC. Efficacy of omalizumab in patients with atopic dermatitis: a systematic review and metaanalysis. J Allergy Clin Immunol 2016; 138: 1719-22.

21. Zink A, Gensbaur A, Zirbs M, et al. Targeting IgE in severe atopic dermatitis with a combination of immunoadsorption and omalizumab. Acta Derm Venereol 2016; 96: 72-6.

22. Hotze M, Baurecht H, Rodriguez E, et al. Increased efficacy of omalizumab in atopic dermatitis patiens with wild-type filaggrin status and higher levels of phosphatidylcholines. Allergy 2014; 69: 132-5.

23. Umetsu DT, Rachid R, Schneider LC. Oral immunotherapy and anti-lgE antibody treatment for food allergy. World Allergy Organ J 2015; 8: 20.

24. Wood RA, Kim JS, Lindblad R, et al. A randomized, doubleblind, placebo-controlled study of omalizumab combined with oral immunotherapy for the treatment of cow's milk allergy. J Allergy Clin Immunol 2016; 137: 1103-10.

25. Labrosse R, Graham F, Des Roches A, Bégin P. The use of omalizumab in food oral immunotherapy. Arch Immunol Ther Exp (Warsz) 2017; 65: 189-99.

26. Wood RA. Oral Immunotherapy for food allergy. J Investig Allergol Clin Immunol 2017; 27: 151-9.

27. Feuille E, Nowak-Węgrzyn A. Oral immunotherapy for food allergies. Ann Nutr Metab 2016; 68: 19-31. 